\title{
Availability and Profit Analysis of 3-out-of-4 Repairable System with Preventive Maintenance
}

\author{
Ibrahim Yusuf \\ Department of Mathematical Sciences, Faculty of Science, Bayero University, \\ Kano, Nigeria \\ Email: ibrahimyusif@yahoo.com
}

\begin{abstract}
Studies on two unit repairable standby system dealing with availability and profit analysis involving preventive maintenance are numerous. However little attention is paid on the study of evaluation of reliability characteristics such as availability, busy period and profit function of multi component system such as 3-out-of-4 system involving four types of failures. In this paper, we studied the availability and profit analysis of a repairable redundant 3-out-of-4 system with preventive maintenance involving four types of failures and develop explicit expressions for steady-state availability and profit function for the system using Chapman-Kolmogorov equations. Some particular cases have also been obtained analytically and graphically to see the impact of preventive maintenance on some system measures of effectiveness .Certain important result have been evaluated as special cases. Results have shown that system with preventive maintenance is better in terms of system effectiveness than system without preventive maintenance.
\end{abstract}

Keywords: Availability, Profit, redundancy, preventive maintenance, busy period

\section{Introduction}

Studies on redundant system are becoming more and richer day by day due to the fact that numbers of researchers in the field of reliability of redundant system are making huge contributions. Models of redundant systems as well as methods of evaluating system reliability indices such as mean time to system failure (MTSF), 
system availability, busy period of repairman, profit analysis, etc have been studied in order to improve the system effectiveness.

There are systems of three/four units in which two/three units are sufficient to perform the entire function of the system. Example of such systems are 2-out-of3,2-out-of-4, or 3-out-of-4 redundant systems. These systems have wide application in the real world. The communication system with three transmitters can be sited as a good example of 2-out-of-3 redundant system. Many research results have been reported on reliability of 2-out-of-3 redundant systems. For example, Chander and Bhardwaj[1], analyzed reliability models for 2-out-of-3 redundant system subject to conditional arrival time of the server. Chander and Bhardwaj [2] present reliability and economic analysis of 2-out-of-3 redundant system with priority to repair. Bhardwaj and Malik [3] studied MTSF and cost effectiveness of 2-out-of-3 cold standby system with probability of repair and inspection.

El-Said [5] and Haggag [6] examined the cost analysis of two unit cold standby system involving preventive maintenance respectively. Wang et al [4] examined the cost benefit analysis of series systems with cold standby components and repairable service station. Haggag [7] analyzed cost analysis of repairable k-outof-n system with dependable failures and standby support. Wang and Kuo [8] studied the cost and probabilistic analysis of series system with mixed standby components. Wang et al [9] studied cost benefit analysis of series systems with warm standby components involving general repair time where the server is not subject to breakdowns.

This study is an extension of the work of El-Said [5] and Haggag [6]. The system is attended by one repairman. In this study, we developed the explicit expressions for availability and profit function and perform simulations to see the behavior of the system and comparison is perform through simulations.

\subsection{Notations}

$\alpha_{i} \quad$ Constant repair rates for type $i=1,2,3,4$

$\beta_{i} \quad$ Constant failure rates for types $i=1,2,3,4$

$\mu \quad$ Constant rate end of preventive maintenance

$\lambda$ Constant rate of taking the unit into preventive maintenance

$A \quad$ System transition rate matrix

$A_{1}(\infty)=A V 1$ System availability as time $t \rightarrow \infty$

$A_{2}(\infty)=A V 2$ System availability as time $t \rightarrow \infty$ when there is no preventive maintenance

$B_{1}(\infty)$ Busy period of repairman as $t \rightarrow \infty$

$B_{2}(\infty)$ Busy period of repairman as $t \rightarrow \infty$ when there is no preventive maintenance

$F(\infty)$ Expected frequency of preventive maintenance as $t \rightarrow \infty$

$P$ Stationary probabilities vector given by 
$P=\left[P_{1}, P_{2}, P_{3}, P_{4}, P_{5}, P_{6}, P_{7}, P_{8}, P_{9}, P_{10}\right]^{T}$

$P_{i} \quad$ State probabilities

$P F_{1}$ Profit function of the system

$\mathrm{PF}_{2} \quad$ Profit function of the system without preventive maintenance

\subsection{Assumptions}

- The system is 3-out-of-4 system

- The system can be in Operation, Fail state or preventive maintenance

- The system suffer four types of failures

- The system is down when number of units failure goes beyond one

- Failure rates and repairs follow exponential

- Failure rates and repair rates are constant

- The system is attended by one repairman

\section{Markov Modeling of the System}

In this section, the 3-out-of-4 redundant system is described. Through Markov assumption, the Chapman-Kolmogorov's equations are obtained for the analysis of state probabilities. The system comprise of four units in which at least three units most be in operational for the system to work. Malfunctioning of two units above lead the system to go down. The units can work consecutively or randomly as can be seen in the states of the system given below. The states of the system according Markov chain is shown in Fig. 1 below.

State 0: initial state, all the three units work, one unit in standby, and the system is working

State 1: units 1, 2, and 4 are working; unit 3 is down and under repair, and the system is working

State 2: units 1, 3 and 4 are working, unit 2 is down and under repair, and the system is working

State 3: units 2, 3 and 4 are working, unit 1 is down and under repair, and the system is working

State 4: units one is down, under repair, units 2 is down and waiting for repair, units 3 and 4 are good, and the system failed

State 5: unit 1 is down, under repair, unit 3 is down, waiting for repair, units 2 and 4 are good, and the system failed

State 6: unit 1 and 2 are good, unit 3 is down, and waiting for repair, unit 4 is down, under repair, and the system failed

State 7: unit 1 is down, waiting for repair, units 2 and 3 are good, unit 4 is down, under repair, and the system failed 
State 8: units 1 and 4 are good, unit 2 is down, and waiting for repair, unit 3 is down, under repair, and the system failed

State 9: units 1 and 3 are good, unit 2 is down, and waiting for repair, unit 4 is down, under repair, and the system failed

State 10: all the units are under preventive maintenance, and the system is working

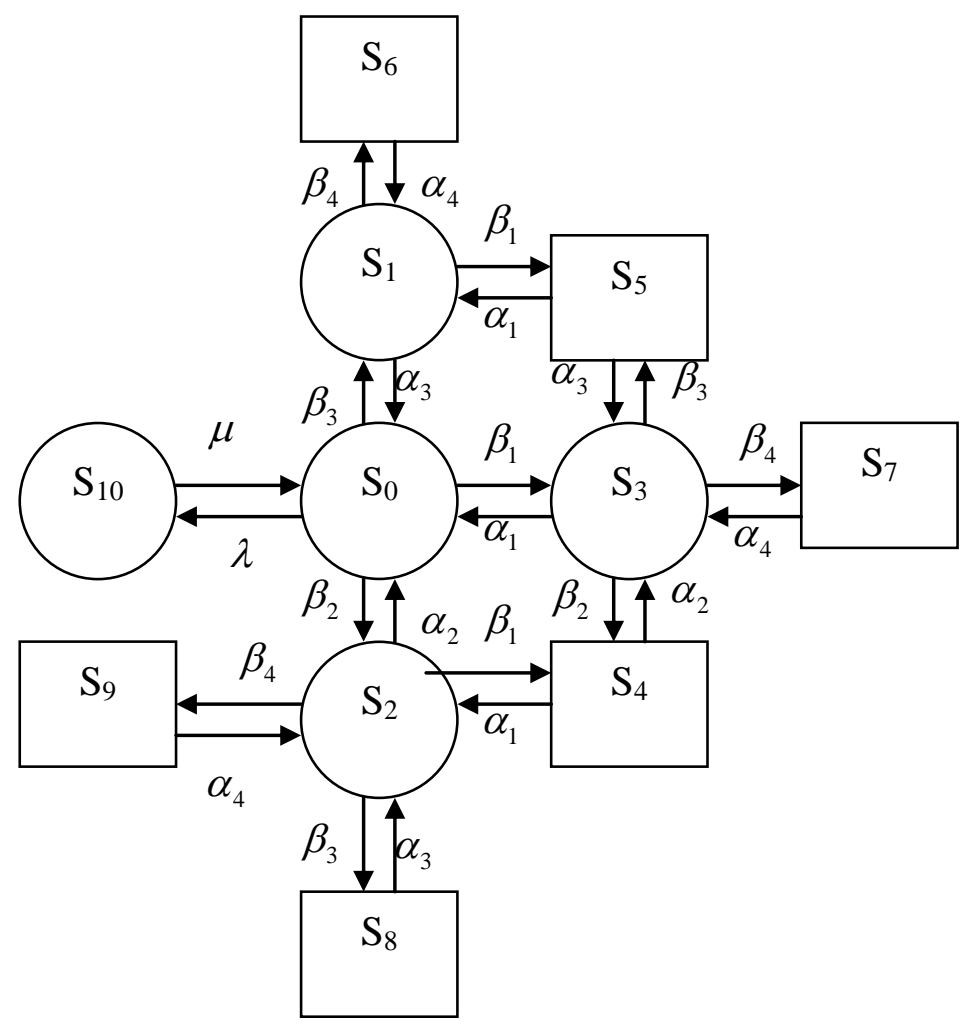

Fig. 1: schematic diagram of the system

\subsection{Availability Analysis for System}

Let $P_{i}(t)$ be the probability that the system is in state $i$ at time $t$. The corresponding set of kolmogorov's differential equations is:

$$
\begin{aligned}
& P_{0}^{\prime}(t)=-\left(\beta_{1}+\beta_{2}+\beta_{3}+\lambda\right) P_{0}(t)+\alpha_{3} P_{1}(t)+\alpha_{2} P_{2}(t)+\alpha_{1} P_{3}(t)+\mu P_{10}(t) \\
& P_{1}^{\prime}(t)=-\left(\alpha_{3}+\beta_{1}+\beta_{2}+\beta_{4}\right) P_{1}(t)+\beta_{3} P_{0}(t)+\alpha_{1} P_{5}(t)+\alpha_{4} P_{6}(t)+\alpha_{2} P_{8}(t) \\
& P_{2}^{\prime}(t)=-\left(\alpha_{2}+\beta_{1}+\beta_{3}+\beta_{4}\right) P_{2}(t)+\beta_{2} P_{0}(t)+\alpha_{1} P_{4}(t)+\alpha_{3} P_{8}(t)+\alpha_{4} P_{9}(t) \\
& P_{3}^{\prime}(t)=-\left(\alpha_{1}+\beta_{2}+\beta_{3}+\beta_{4}\right) P_{3}(t)+\beta_{1} P_{0}(t)+\alpha_{2} P_{4}(t)+\alpha_{3} P_{5}(t)+\alpha_{4} P_{7}(t) \\
& P_{4}^{\prime}(t)=-\left(\alpha_{1}+\alpha_{2}\right) P_{4}(t)+\beta_{1} P_{2}(t)+\beta_{2} P_{3}(t) \\
& P_{5}^{\prime}(t)=-\left(\alpha_{1}+\alpha_{3}\right) P_{5}(t)+\beta_{1} P_{1}(t)+\beta_{3} P_{3}(t)
\end{aligned}
$$


$P_{6}^{\prime}(t)=-\alpha_{4} P_{6}(t)+\beta_{4} P_{1}(t)$

$P_{7}^{\prime}(t)=-\alpha_{4} P_{7}(t)+\beta_{4} P_{3}(t)$

$P_{8}^{\prime}(t)=-\left(\alpha_{2}+\alpha_{3}\right) P_{8}(t)+\beta_{2} P_{1}(t)+\beta_{3} P_{2}(t)$

$P_{9}^{\prime}(t)=-\alpha_{4} P_{9}(t)+\beta_{4} P_{2}(t)$

$P_{10}^{\prime}(t)=-\mu P_{10}(t)+\lambda P_{0}(t)$

For the availability case of Fig. 1 following Wang et al [4], El-said [5] and Haggag [6], the initial conditions for this system are:

$$
\begin{aligned}
& P(0)=\left[P_{1}(0), P_{2}(0), P_{3}(0), P_{4}(0), P_{5}(0), P_{6}(0), P_{7}(0), P_{8}(0), P_{9}(0), P_{10}(0)\right]= \\
& {[1,0,0,0,0,0,0,0,0,0,0]}
\end{aligned}
$$

From the states of the system describes above, it is possible to identify which state is working and which state is failure. Through the state transition diagram in Fig. 1 above, we can obtain the availability of the system. This is done by solving the Chapman-Kolmogorov' equation in (1) to evaluate the state probabilities. Computation of state probabilities involves solving the normalized Chapmankolmogorov system linear differential equations:

$A P=0$

Subject to $\sum_{i=0}^{10} P_{i}(\infty)=1$

Following Wang et al [4], El-Said [5] and Haggag [6] the system availability can be obtained from the solutions for $P_{i}(t), i=0,1,2, \ldots, 10$. States $0,1,2,3$ and 10 in Fig. 1 above are the only working, putting (4) in one of the redundant rows of (3) the system availability is given by:

$$
\begin{aligned}
& A_{1}(\infty)=P_{0}(\infty)+P_{1}(\infty)+P_{2}(\infty)+P_{3}(\infty)+P_{10}(\infty)=\frac{N_{1}}{D_{1}} \\
& N_{1}=\alpha_{1} \alpha_{2} \alpha_{3} \alpha_{4} \mu+\alpha_{1} \alpha_{2} \alpha_{4} \beta_{3} \mu+\alpha_{1} \alpha_{3} \alpha_{4} \beta_{2} \mu+\alpha_{2} \alpha_{3} \alpha_{4} \beta_{1} \mu+\alpha_{1} \alpha_{2} \alpha_{3} \alpha_{4} \lambda \\
& D_{1}=\alpha_{1} \alpha_{2} \alpha_{3} \alpha_{4} \mu+\alpha_{1} \alpha_{2} \alpha_{4} \beta_{3} \mu+\alpha_{1} \alpha_{2} \alpha_{3} \alpha_{4} \lambda+\alpha_{1} \alpha_{2} \beta_{3} \beta_{4} \mu+\alpha_{1} \alpha_{3} \alpha_{4} \beta_{2} \mu \\
& \quad+\alpha_{1} \alpha_{3} \beta_{2} \beta_{4} \mu+\alpha_{1} \alpha_{4} \beta_{2} \beta_{3} \mu+\alpha_{2} \alpha_{3} \alpha_{4} \beta_{1} \mu+\alpha_{2} \alpha_{3} \beta_{1} \beta_{4} \mu+\alpha_{2} \alpha_{4} \beta_{1} \beta_{3} \mu+\alpha_{3} \alpha_{4} \beta_{1} \beta_{2} \mu
\end{aligned}
$$

\subsection{Busy period analysis}

Units failure can be observed in states $2,3,4, \ldots, 9$ from the states of the system described above. In these states, it can be observed that the available repairman is busy repairing the failed unit. Where there is more than one unit failure, one unit 
will be under repair and the one unit is waiting for repair while the remaining unit are good and stay as standby. The repair is therefore busy in the states $2,3,4, \ldots, 9$

From equation (3) and (4) using the same initial condition in (2) above , the busy period is given by:

$$
\begin{aligned}
& B_{1}(\infty)=1-\left[P_{0}(t)+P_{10}(t)\right]=\frac{N_{2}}{D_{1}} \\
& \begin{aligned}
N_{2} & =\alpha_{1} \alpha_{2} \alpha_{4} \beta_{3} \mu+\alpha_{1} \alpha_{2} \beta_{3} \beta_{4} \mu+\alpha_{1} \alpha_{3} \alpha_{4} \beta_{2} \mu+\alpha_{1} \alpha_{3} \beta_{2} \beta_{4} \mu+\alpha_{1} \alpha_{4} \beta_{2} \beta_{3} \mu+\alpha_{2} \alpha_{3} \alpha_{4} \beta_{1} \mu \\
& +\alpha_{2} \alpha_{3} \beta_{1} \beta_{4} \mu+\alpha_{2} \alpha_{4} \beta_{1} \beta_{3} \mu+\alpha_{3} \alpha_{4} \beta_{1} \beta_{2} \mu
\end{aligned}
\end{aligned}
$$

\subsection{Expected frequency of Frequency of preventive maintenance}

The expected frequency of preventive maintenance per unit in steady state is given by:

$$
F(\infty)=P_{10}(\infty)=\frac{N_{3}}{D_{1}}
$$

The state probability $P_{10}(\infty)$ is obtained by substituting (4) in one of the redundant rows of (3). Thus

$$
N_{3}=\alpha_{1} \alpha_{2} \alpha_{3} \alpha_{4} \lambda
$$

\subsection{Profit analysis}

The units are subjected to repairs as can be observed in state 2, 3,4,5,6,7,8 and 9 from Fig. 1 the repairman performed repairs to failed units in state 2,3,4,5,6,7,8 and 9. Let $C_{0}, C_{1}$ and $C_{2}$ be the revenue generated when the system is in working state and no income when in failed state, cost of each repairs and cost per preventive maintenance. Following El-said [5] and Haggag [6] the expected profit per unit time incurred to the system in the steady-state is given by:

$$
P F_{1}=C_{0} A(\infty)-C_{1} B(\infty)-C_{2} F(\infty)
$$

Where $P F_{1}$ : is the profit incurred to the system

$C_{0}:$ is the revenue per unit up time of the system

$C_{1}$ : is the cost per unit time which the system is under repair

$C_{2}$ : is the cost due to preventive maintenance

\subsection{Special case when preventive maintenance is not allowed:}

Through the state transition diagram in Fig. 1 above, we can obtain the availability of the system. This is done by solving the Chapman-Kolmogorov' 
equation in (1) to evaluate the state probabilities when there is no preventive maintenance. Thus there is no state 10 .

Computation of state probabilities involves solving the normalized Chapmankolmogorov system linear differential equations:

$A P=0$

Subject to $\sum_{i=0}^{9} P_{i}(\infty)=1$

Where $P=\left[P_{0}, P_{1}, P_{2}, P_{3}, P_{4}, P_{5}, P_{6}, P_{7}, P_{8}, P_{9}\right]^{T}$

The steady-state availability when preventive maintenance is not allowed is given by :

$$
\begin{aligned}
& A_{2}(\infty)=P_{0}(\infty)+P_{1}(\infty)+P_{2}(\infty)+P_{3}(\infty)=\frac{N_{4}}{D_{2}} \\
& N_{4}=\alpha_{1} \alpha_{2} \alpha_{3} \alpha_{4}+\alpha_{1} \alpha_{2} \alpha_{4} \beta_{3}+\alpha_{1} \alpha_{3} \alpha_{4} \beta_{2}+\alpha_{2} \alpha_{3} \alpha_{4} \beta_{1} \\
& D_{2}=\alpha_{1} \alpha_{2} \beta_{3} \beta_{4}+\alpha_{1} \alpha_{2} \alpha_{3} \alpha_{4}+\alpha_{1} \alpha_{2} \alpha_{4} \beta_{3}+\alpha_{1} \alpha_{3} \alpha_{4} \beta_{2}+\alpha_{1} \alpha_{4} \beta_{2} \beta_{3}+\alpha_{1} \alpha_{3} \beta_{2} \beta_{4} \\
& \quad+\alpha_{2} \alpha_{4} \beta_{1} \beta_{3}+\alpha_{2} \alpha_{3} \beta_{1} \beta_{4}+\alpha_{2} \alpha_{3} \alpha_{4} \beta_{1}+\alpha_{3} \alpha_{4} \beta_{1} \beta_{2}
\end{aligned}
$$

In the steady state, the derivatives of the state probabilities become zero this will enable us to compute steady state busy when preventive maintenance is not allowed. The steady state busy period $B_{2}(\infty)$ is therefore:

$$
\begin{aligned}
& B_{2}(\infty)=1-P_{0}(\infty)=\frac{N_{5}}{D_{2}} \\
& \begin{aligned}
N_{5} & =\alpha_{1} \alpha_{2} \beta_{3} \beta_{4}+\alpha_{1} \alpha_{2} \alpha_{4} \beta_{3}+\alpha_{1} \alpha_{3} \alpha_{4} \beta_{2}+\alpha_{1} \alpha_{4} \beta_{2} \beta_{3}+\alpha_{1} \alpha_{3} \beta_{2} \beta_{4} \\
& +\alpha_{2} \alpha_{4} \beta_{1} \beta_{3}+\alpha_{2} \alpha_{3} \beta_{1} \beta_{4}+\alpha_{2} \alpha_{3} \alpha_{4} \beta_{1}+\alpha_{3} \alpha_{4} \beta_{1} \beta_{2}
\end{aligned}
\end{aligned}
$$

The units are subjected to repairs as can be observed in state 2, 3,4,5,6,7,8 and 9 from Fig. 1 the repairman performed repairs to failed units in state 2,3,4,5,6,7,8 and 9. Let $C_{0}$ and $C_{1}$ be the revenue generated when the system is in working state and no income when in failed state and cost of each repair (corrective maintenance), a $[1,2,4]$. Following $[1,2]$ the expected total profit per unit time incurred to the system in the steady-state when preventive maintenance is not allowed is

$$
P F_{2}=C_{0} A_{2}(\infty)-C_{1} B_{2}(\infty)
$$

Where $P F_{2}$ : is the profit incurred to the system 
$C_{0}:$ is the revenue per unit up time of the system

$C_{1}$ : is the cost per unit time which the system is under repair

\section{Graphical Study of System Behavior}

Graphs were plotted in figures 2 to 5 for the availability and expected profit with respect to both $\alpha_{1}$ and $\beta_{1}$. Fig. 2 shows the effect of $\alpha_{1}$ on expected profit; Fig. 3 shows the effect of $\alpha_{1}$ on system availability, Fig. 4 shows the effect of $\beta_{1}$ on system availability while Fig. 5 shows the effect of $\beta_{1}$ on expected profit.

For simulation in Fig. 2and Fig. 3 we fixed $C_{0}=1000, C_{1}=100$ and $C_{2}=50$ for Fig. 2 and fixed $\alpha_{2}=0.6, \quad \alpha_{3}=0.7 \quad, \quad \alpha_{4}=0.9 \quad, \quad \beta_{1}=0.2 \quad$, $\beta_{2}=0.1, \beta_{3}=0.3, \beta_{4}=0.5, \mu=0.8$ and $\lambda=0.05$ and vary $\alpha_{1}$ for both Fi2. and Fig. 3. The simulations in Fig. 2 have shown that the expected profits for both systems with and without preventive maintenance increase with increase in type I repair rate $\alpha_{1}$. It is clear from Fig. 2 that the expected profit of system with preventive maintenance increases slightly with respect to type I repair rate $\alpha_{1}$ than the expected profit of system without preventive maintenance. In Fig.3, it is clear that the system availability increases for both systems with and without preventive maintenance. Here also the system availability of system with preventive maintenance increases slightly than system availability of system without preventive maintenance.

For simulation in Fig.4 we fixed: $\alpha_{1}=0.01, \quad \alpha_{2}=0,02, \quad \alpha_{3}=0.03$, $\alpha_{4}=0.001, \quad \beta_{2}=0.1, \quad \beta_{3}=0.3, \beta_{4}=0.5, \mu=0.04, \lambda=0.05$, and vary $\beta_{1}$. The simulations have shown that system availability decreases for both systems with and without preventive maintenance with respect to type I failure rate $\beta_{1}$. The system availability for the system without preventive maintenance decrease slightly than the system with preventive maintenance.

Fig. 5 provides the comparison of profit functions for the systems with and without preventive maintenance, we fixed $C_{0}=1000, C_{1}=100, C_{2}=50$, $\alpha_{1}=0.7, \quad \alpha_{2}=0.9, \quad \alpha_{3}=0.8$ $\alpha_{4}=0.9, \beta_{2}=0.1, \beta_{3}=0.3, \beta_{4}=0.5, \mu=0.9, \lambda=0.05$ and vary $\beta_{1}$. The simulation have shown that expected profit decreases for both systems with and without preventive maintenance with respect to type I failure rate $\beta_{1}$. The expected profit for the system without preventive maintenance decrease slightly than the expected profit of the system with preventive maintenance. 


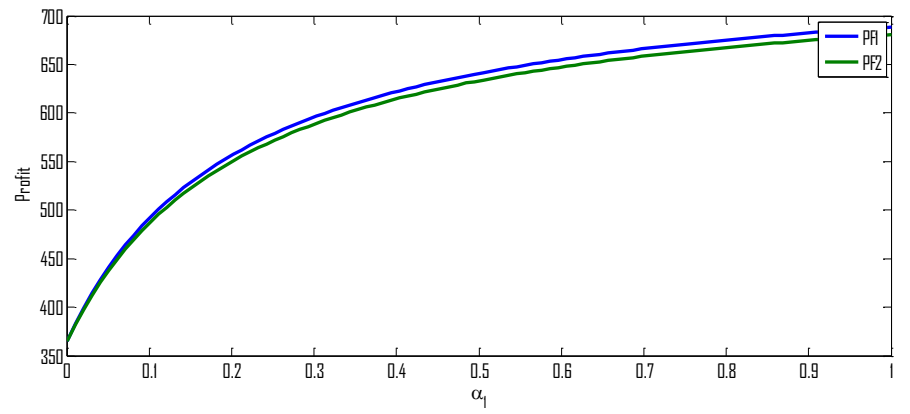

Fig.2: effect of $\alpha_{1}$ on expected profit

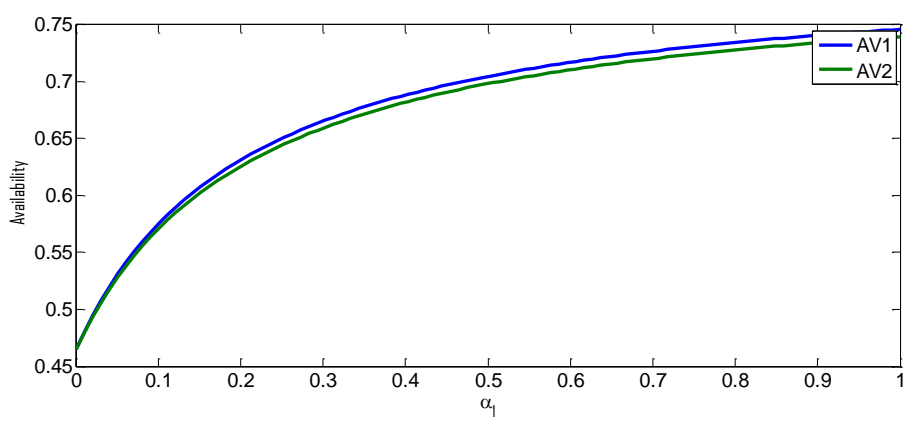

Fig. 3: effect of $\alpha_{1}$ on system availability

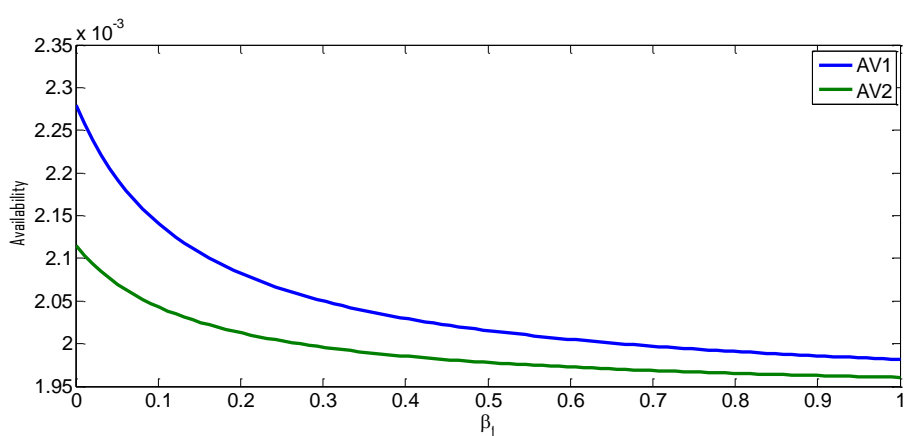

Fig. 4: effect of $\beta_{1}$ on system availability

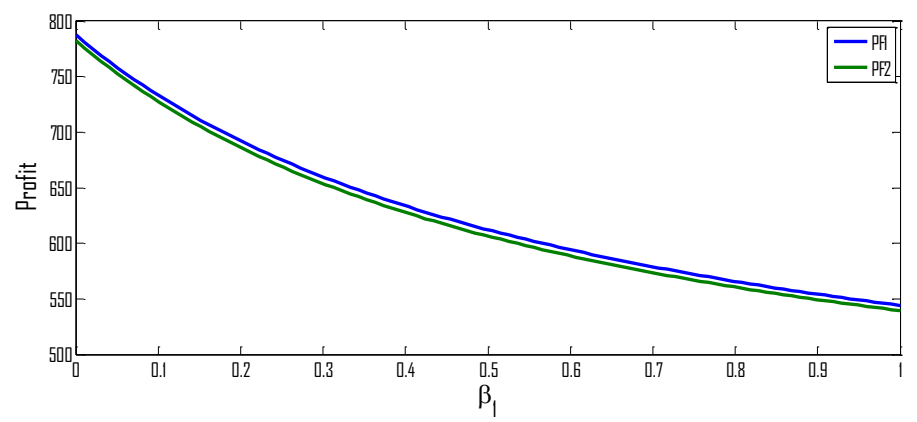

Fig. 5: effect of $\beta_{1}$ on expected profit 


\section{Conclusion}

In this study, we developed the explicit expressions for availability and profit function of 3-out-of-4 repairable system and perform comparative analysis. Through the analysis, we conclude that system with preventive maintenance is more effective than system without preventive maintenance.

\section{Acknowledgement}

The author would like to thank the editor and the reviewers for their suggestions which have improved the manuscript.

\section{References}

[1] Bhardwj, R.K. and Chander, S. (2007). Reliability and cost benefit analysis of 2-out-of-3 redundant system with general distribution of repair and waiting time. DIAS- Technology review- An Int. J. of business and IT. 4(1), 28-35

[2] Chander, S. and Bhardwaj, R.K. (2009). Reliability and economic analysis of 2-out-of-3 redundant system with priority to repair. African J. of Maths and comp. sci, 2(11), 230-236.

[3] Bhardwj,R.K., and S.C. Malik. (2010). MTSF and Cost effectiveness of 2out-of-3 cold standby system with probability of repair and inspection. Int. J. of Eng. Sci. and Tech. 2(1), 5882-5889

[4] Wang., k. Hsieh., C. and Liou, C (2006). Cost benefit analysis of series systems with cold standby components and a repairable service station. Journal of quality technology and quantitative management, 3(1), 77-92

[5] El-Said, K.M.,. (2008).Cost analysis of a system with preventive maintenance by using Kolmogorov's forward equations method. American Journal of Applied Sciences 5(4), 405-410

[6] Haggag, M.Y., (2009). Cost analysis of a system involving common cause failures and preventive maintenance. Journal of Mathematics and Statistics 5(4), 305-310

[7] Haggag, M.Y., (2009). Cost analysis of k-out-of-n repairable system with dependent failure and standby support using Kolmogorov's forward equations method .Journal of Mathematics and Statistics 5(4), 401-407.

[8] Wang, K.H and Kuo, C.C. (2000). Cost and probabilistic analysis of series systems with mixed standby components. Applied Mathematical Modelling, 24, 957-967

[9] Wang, K.C., Liou, Y.C, and Pearn W. L. (2005). Cost benefit analysis of series systems with warm standby components and general repair time. ,Mathematical Methods of operation Research, 61, 329-343. 